\title{
Concomitant medullary thyroid carcinoma with paraganglioma-like pattern and papillary thyroid carcinoma
}

\author{
C Greco ${ }^{1,2}$, G Brigante 1,2 , E Taliani², S Corrado33, M Simoni1,2 and B Madeo²
}

1 Unit of Endocrinology, Department of Biomedical, Metabolic and Neural Sciences, University of Modena and Reggio Emilia, Modena, Italy, 2Unit of Endocrinology, Department of Medical Specialties, Azienda Ospedaliero-Universitaria di Modena, Ospedale Civile di Baggiovara, Modena, Italy, and 3Department of Diagnostic, Clinical Medicine and Public Health, Azienda Ospedaliero-Universitaria di Modena, Modena, Italy

Correspondence should be addressed to G Brigante

Email

giulia.brigante@unimore.it

\section{Summary}

A 74-year-old man was referred to the Endocrinology Unit because of multinodular goiter. The dominant nodule $(1.7 \times 1.9 \times 2.4 \mathrm{~cm})$, at the medium-superior third of the left lobe, was inhomogeneously hypoechoic, with irregular margins, macrocalcifications and intranodular vascularization. Fine-needle aspiration biopsy (FNAB) was performed. The cytological diagnosis was TIR 2, benign, according to the 2013 Italian thyroid cytology classification system. Moderately high serum calcitonin (s-Ct) $(61.5 \mathrm{pg} / \mathrm{mL}$, n.r. 0-7.5) and normal CEA were detected. The Ct level in FNAB wash-out fluid (Ct-FNAB) was $1450 \mathrm{pg} / \mathrm{mL}$. Based on s-Ct and Ct-FNAB levels, patient underwent total thyroidectomy. Macroscopically, a dominant circumscribed nodule of $2 \mathrm{ecm}$ was described; the histological and immunohistochemical features identified medullary thyroid carcinoma (MTC) with paraganglioma (PG)-like pattern positive for Ct, CEA and chromogranin and negative for S-100 sustentacular cells (SC). Moreover, papillary carcinoma of $3 \mathrm{~mm}$ in the right lobe was also associated. No areas of hyperaccumulation of the tracer were documented at Ga68 PET/CT. No RET-proto-oncogene mutations were found. Post-surgery s-Ct levels were within normal range $(4 \mathrm{pg} / \mathrm{mL})$. Two years after thyroidectomy, the patient is still disease-free. We reported a case of sporadic and rare variant of MTC: this is the ninth described case of PG-like MTC. In this case, cytologically benign, the clinical suspicion arose from high $\mathrm{Ct}$ values at FNAB wash-out fluid. Even if clinical behavior of this variant seems indolent, additional studies are necessary to understand prognoses and predictive factors.

\section{Learning points:}

- Several unusual histological variants of medullary thyroid carcinoma (MTC) have been described such as spindle cell, giant cell, clear cell, melanotic, squamous, angiosarcoma-like variants; even rarer is the paraganglioma (PG)like pattern.

- We here describe a case of medullary PG-like thyroid carcinoma in a 74-year-old man. This is a rare histological variant of MTC hardly diagnosed by cytology, since immunohistochemical investigations are necessary.

- Measurement of calcitonin both in serum and in wash-out fluid from fine-needle aspiration could be an additional tool for an early and non-invasive identification of these variants.

\section{Background}

Medullary thyroid carcinoma (MTC) is a rare, welldifferentiated neuroendocrine neoplasm of thyroid, derived from the parafollicular calcitonin (Ct)-producing cells (C-cells) and accounts for 5-8\% of all thyroid malignancies. Up to $75 \%$ of MTC cases occur sporadically. Genetical forms of MTC show an autosomal dominant 
pattern of trasmission. RET oncogene mutations in neural crest tissue in thyroid can lead to MTC development (1).

Elevated serum $\mathrm{Ct}(\mathrm{sCt})$ is a very sensitive and specific marker of MTC, although some pathological conditions can be accompanied by a slight increase of sCt. Moreover, sCt has proved to be highly sensitive for prognostic assessment, follow-up, and evaluation of treatment response in MTC. However, some MTC cases with normal sCt have been reported (2). Macroscopically, MTC is a solid, non-capsulated but well-circumscribed, neoplasm usually located in the middle third of the thyroid lobe, where C cells are concentrated. Microscopically, MTC is characterized by nests of round, ovoid, polygonal, plasmacytoid or splindle-shaped cells. There is no follicle development as the tumor derives from parafollicular C cells. MTC cells may infiltrate structures surrounding thyroid and give metastases to the cervical, mediastinal, and distant organs, such as lung, liver, and skeletal muscle (1).

Several unusual histological variants of MTC have been described such as spindle cell, giant cell, clear cell, melanotic, squamous, angiosarcoma-like variants. Even rarer is the variant showing a paraganglioma (PG)-like pattern, composed of cells with a broad trabecular pattern. Immunohistochemically, the PG-like MTC is negative for thyroglobulin and shows strong $\mathrm{Ct}$, carcinoembryonic antigen (CEA), cromogranin $\mathrm{A}$ and synaptophysin positivity (1).

We herein report a recent case of PG-like MTC compared to previous cases in literature.

\section{Case presentation}

A 74-year-old Italian man was referred to the Endocrinology Unit in March 2017 because of multinodular goiter. He was on antihypertensive and anticoagulant therapy for hypertension and chronic atrial fibrillation. No family history of thyroid cancer or multiple endocrine neoplasia was reported. Physical examination revealed a mass in the left lobe of thyroid gland. Moderately high values of sCt $(61.5 \mathrm{pg} / \mathrm{mL}$, normal range: 0-12 pg/mL) were detected. Serum CEA and parathormone, and urinary metanephrine were normal. The patient had normal thyroid function (thyroid-stimulating hormone, TSH: $2.08 \mu \mathrm{IU} / \mathrm{mL}$, normal range: 0.35-4.94) and no alteration of calcium metabolism (serum calcium: 9.9 $\mathrm{mg} / \mathrm{dL}$, normal range: 8.5-10.5; phosphorus: $3.1 \mathrm{mg} / \mathrm{dL}$, normal range: 2.5-5.1; albumin: $4 \mathrm{~g} / \mathrm{dL}$, normal range: 3.5-5). At ultrasound, thyroid showed an inhomogeneous echotexture and presented at the medium-superior third of the left lobe a voluminous nodule $(1.7 \times 1.9 \times 2.4 \mathrm{~cm})$ inhomogeneously hypoechoic with irregular margins, macrocalcifications and intranodular vascularization at Doppler evaluation (Fig. 1); there was also a small $(0.7 \times 0.6 \times 0.8 \mathrm{~cm})$ cystic nodule in the right lobe. There was no cervical lymphadenopathy.

\section{Investigation}

At ultrasound evaluation, the dominant nodule was classified as highly suspected for malignancy, and then fine-needle aspiration biopsy (FNAB) was performed. The cytological diagnosis was benign based on hematic and colloidal material comprising hyperplastic nests of thyrocytes, mostly small (TIR 2 according to the Italian thyroid cytology classification system) (Fig. 2). The Ct measurement in FNAB wash-out fluid (Ct-FNAB) was high (1450 pg/mL). Based on serum- and Ct-FNAB results, despite benign cytology, patient underwent total thyroidectomy with central compartment lymph node dissection.

\section{Treatment}

After total thyroidectomy and lymph node dissection, the surgical specimen showed macroscopically a circumscribed nodule of $2 \mathrm{~cm}$ in greatest diameter in the left thyroid lobe. Histopathological examination identified MTC with pattern typical of paragangliomas

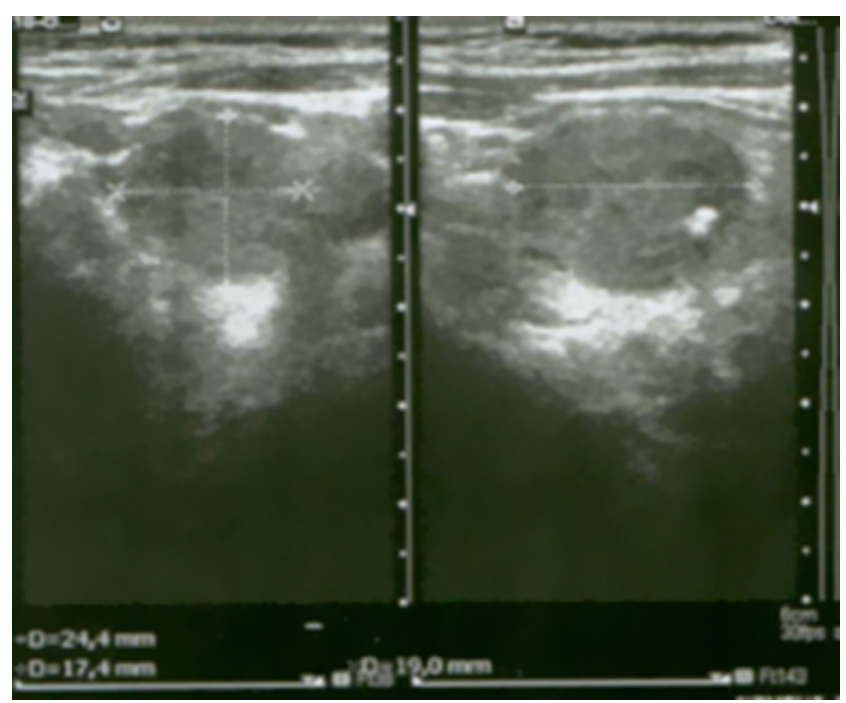

Figure 1

Thyroid ultrasound. Nodule inhomogeneously hypoechoic with irregular margins, macrocalcifications and intranodular vascularization at Doppler evaluation at the medium-superior third of the left lobe $(17.4 \times 19 \times 24.4 \mathrm{~mm})$. 


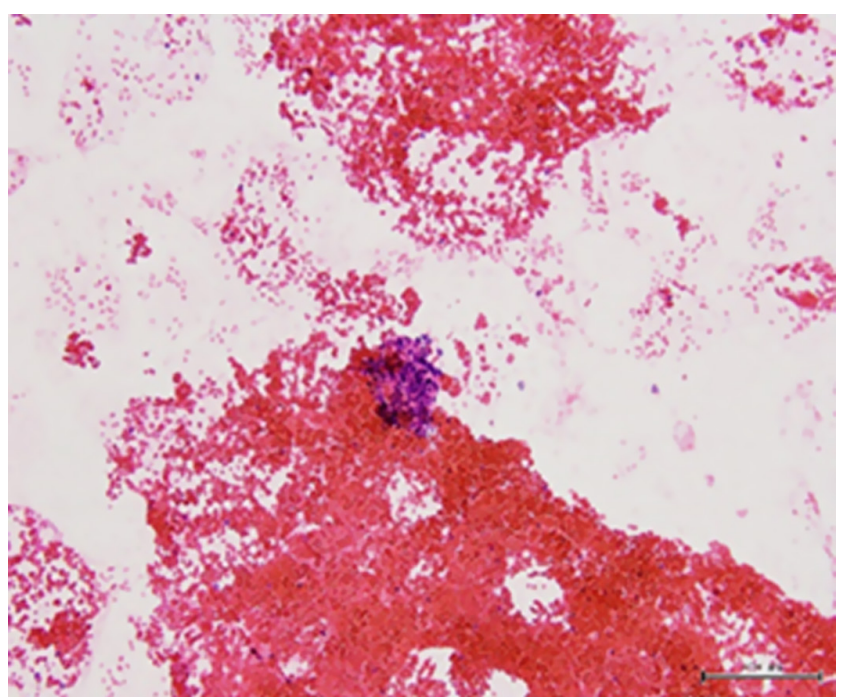

Figure 2

Cytological analysis of dominant nodule. Hematic and colloidal material comprising hyperplastic nests of thyrocytes, mostly small: the cytological diagnosis was TIR 2, benign, according to Italian thyroid cytology classification system.

of $2 \mathrm{~cm}$ (Fig. 3). Immunohistochemical study showed diffuse positive reaction for Ct, CEA and chromogranin, and focal positive reaction for thyroid transcription factor-1 (TTF-1). However, S-100 stain did not reveal the sustentacular cells peculiar to benign paragangliomas (Fig. 4). In the right lobe histological section, papillary thyroid carcinoma (PTC) of $3 \mathrm{~mm}$, with minimal extension to the peri-thyroid adipose tissue was also associated. There
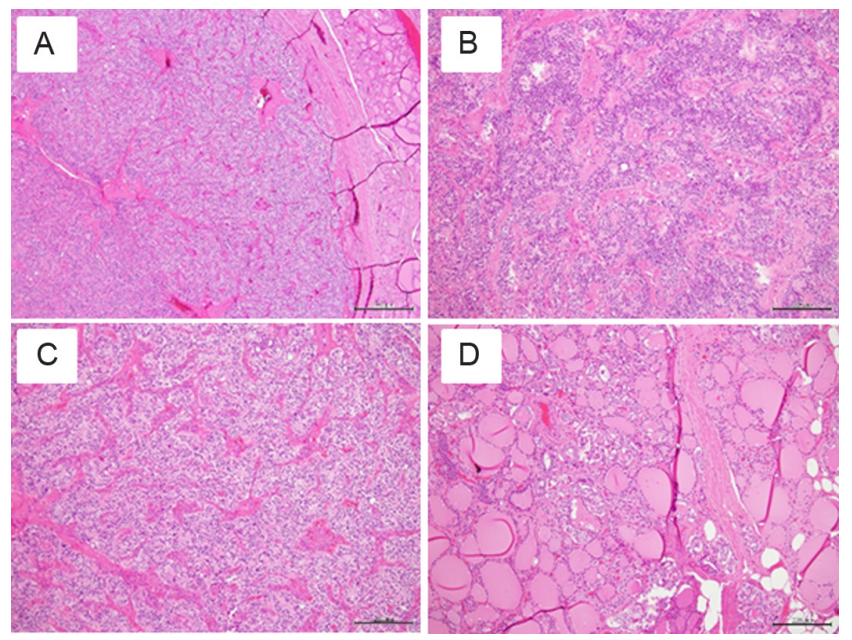

Figure 3

Histological analysis. Macroscopically, the surgical specimen showed a circumscribed nodule of $2 \mathrm{~cm}$ in greatest diameter in the left thyroid lobe. Histological features of the present case: sections of tumor showing MTC with pattern typical of paragangliomas (original magnification, $\times 4$ for $A$ and $B, \times 10$ for $C$ and $D$ ).

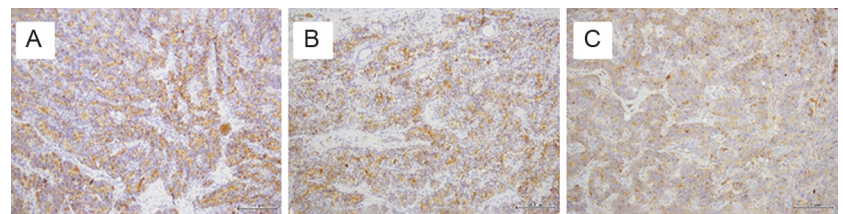

\section{Figure 4}

Immunohistochemical analysis. Immunohistochemical examination of the cytoblock tissue showed diffuse expression of calcitonin (A), CEA (B) and chromogranin $(C)$. The analysis showed negative reaction for protein S-100.

was no focus of C-cell hyperplasia in the adjacent thyroid gland and no evidence of cervical adenopathies.

According to TNM Staging System of American Joint Committee on Cancer (AJCC) seventh edition (in use at time of thyroidectomy), tumor staging was pT2 N0 $\mathrm{Mx}$ - Stage II for medullary thyroid carcinoma and pT3 NO MX - Stage III for papillary thyroid cancer. In actual edition (Eighth Edition TNM/AJCC), staging is the same for PG-like medullary carcinoma and pT1a NO MX - Stage I for papillary cancer.

\section{Outcome and follow-up}

For papillary cancer with minimal extension to the perithyroid adipose tissue, recombinant human TSH (rhTSH)aided thyroid ablation with radioiodine I131 (50 mCi) was performed. At the time of radioablation, we observed TSH $0.12 \mu \mathrm{IU} / \mathrm{mL}$ (normal range 0.35-4.94), free thyroxine fT4 $12.5 \mathrm{pg} / \mathrm{mL}$ (normal range 7-15), thyroglobulin $\mathrm{Tg}$ $<0.1 \mathrm{ng} / \mathrm{mL}$ (normal range 1-48) and anti-thyroglobulin antibodies (AbTg) levels $<1 \mathrm{IU} / \mathrm{mL}$ (normal range $0-4)$. Whole body scintigraphy showed no areas of iodine uptake.

Even if not properly indicated, the patient underwent $\mathrm{Ga}^{68}$ PET-CT after surgery and no areas of hyperaccumulation of the tracer and nor any other disease localization were documented. Successfully, sCt levels fell to $4 \mathrm{pg} / \mathrm{mL}$ after surgery, becoming undetectable in 17 months. Two years after thyroidectomy, the patient has no structural or biochemical evidence of recurrent disease.

Finally, no RET-proto-oncogene mutations were found in exons 8, 10, 11, 13, 14, 15 and 16.

\section{Discussion}

We reported a case of rare and sporadic variant of MTC characterized by PG-like pattern in 74-year-old man with concomitant micropapillary thyroid carcinoma. The patient presented high value of serum $\mathrm{Ct}$ and dominant 
hypoechoic nodule at the medium-superior third of the left lobe at US investigation. The cytological examination at FNAB showed no aspects of malignancy, as in most MTC, but Ct-FNAB was $1450 \mathrm{pg} / \mathrm{mL}$. After total thyroidectomy, histopatological features were consistent with PG-like MTC. Immunohistochemical study obviously showed a diffuse positive reaction for Ct, CEA and chromogranin. Importantly, the neoplasm was focally positive for TTF-1 and did not demonstrate S-100-positive sustentacular cells: either features ruled out the diagnosis of benign paraganglioma. The patient showed no lymph node involvement or distant metastasis and post-surgery $\mathrm{Ct}$ levels fell within the normal range. The follow-up checks were performed performed using basal serum calcitonin assays. After 2 years following thyroidectomy, the patient is without evidence of recurrent disease.

Only eight cases of PG-like MTC have been reported so far. We screened PubMed database using PG-like MTC, paraganglioma-like thyroid carcinoma as key words. We selected seven articles and extracted eight case reports of this rare variant of MTC $(3,4,5,6,7,8,9)$ (Table 1$)$.

Considering characteristics and management of the available cases, our case is peculiar because of $\mathrm{Ct}$ measurement in FNAB wash-out fluid. Specifically, this dosage was fundamental to diagnose a medullary pathology, undiagnosed at cytology and suggested by a modest rise in sCt. As known, cytologic examination from FNAB has several pitfalls in MTC diagnosis, not only in PG-like MTC. A recent meta-analysis demonstrated that cytology is able to detect approximately just half of MTC (10). To try to overcome this limit, emerging evidence shows the role of $\mathrm{Ct}$ measurement from FNAB washout liquid, especially in patients with modestly elevated basal sCT (20-100 pg/mL) (11). In our case, no elements suggestive of malignancy were detected at cytologic analysis but Ct-FNAB was $1450 \mathrm{pg} / \mathrm{mL}$. As shown in Table 1 , FNAB was performed only in six cases: four presented cellular atypias, suggestive of malignancy. In particular, Ryska et al. (8) described the cytological findings of MTC PG-like: ovoid epithelial cells arranged in cohesive clusters with sharp margins and significant nuclear atypia with occasional bizarre and/or binucleated cells. No colloid or other material was present in the background; in one of these cases also Ct expression at immunocytochemistry was demonstrated. FNAB wash-out fluid was not performed in none of the previous reported patients.

Moreover, in five cases, sCt was evaluated, resulting almost always above normal range. Collina et al. (3) and Bockhorn et al. (6) also reported subsequent $\mathrm{sCt}$ normalization after total thyroidectomy. A post-surgical persistence of elevated sCt is reported only in the case of Ryska et al. (8), characterized by lymph nodes and distant metastases. Only Bockhorn et al. (6) evaluated CEA, another marker commonly used in the evaluation and follow-up of medullary carcinoma, finding high value.

Macroscopically, MTCs were always localized in the lobes, never in isthmus, and size never exceeded $1 \mathrm{~cm}$. Moreover, the main data reported in literature concern histopathological and immunohistochemical investigations. The authors described pigmented dendritic cells, strongly reminiscent of the sustentacular cells-like (SCs), also called zellballen, usually found in paragangliomas. These cells exhibited immunoreactivity for $\mathrm{Ct}$, chromogranin $\mathrm{A}, \mathrm{CEA}$, cytokeratin and stained for neuron-specific enolase and $\mathrm{S} 100$ protein. The expression of S100 protein has already been described in adrenal pheochromocytoma and extra-adrenal paraganglioma. No SCs with S100 immunostains were visualized by Jayaram et al. (7) and in one case of Ryska et al. (8), as in our case.

Considering the clinical characteristics, three of the eight cases were men and the overall average age at diagnosis was 54.5 years. Finally, only in two cases $(3,8)$ tumor was in the context of a multiple endocrine neoplasia syndrome (MEN 2A). Moreover, only one case showed lymph node involvement, already present at the diagnosis, and distant metastasis at lung (8).

Furthermore, the present case shows the concomitant presence of PTC and MTC. The co-occurrence of these two cancers in the same patient has already been described, even if rare. The hypotheses of a common genetic drive was excluded because of different mutations in the two histological types: mutation of RET gene for MTC and mainly BRAF gene for PTC. The current case shown two distinct nodules with diagnostic features of MTC and of PTC, separated by non-neoplastic thyroid parenchyma. RET gene was tested and resulted not mutated. We do not have any evaluation about BRAF gene mutation in this patient.

In conclusion, we reported a rare histological variant of MTC, PG-like, composed of cells with a broad trabecular pattern. Clinically, our patient was referred to thyroidectomy because of sCt and Ct-FNAB diagnostic for medullary disease, despite normal cytological result. After surgery, histopatological and immunohistochemical features identified PG-like MTC with diffuse positive reaction for Ct, CEA, chromogranin, focal positive reaction for TTF-1 and negative for S-100 protein. This rare variant of MTC is hardly diagnosed by cytology, since immunohistochemical investigations are necessary. 


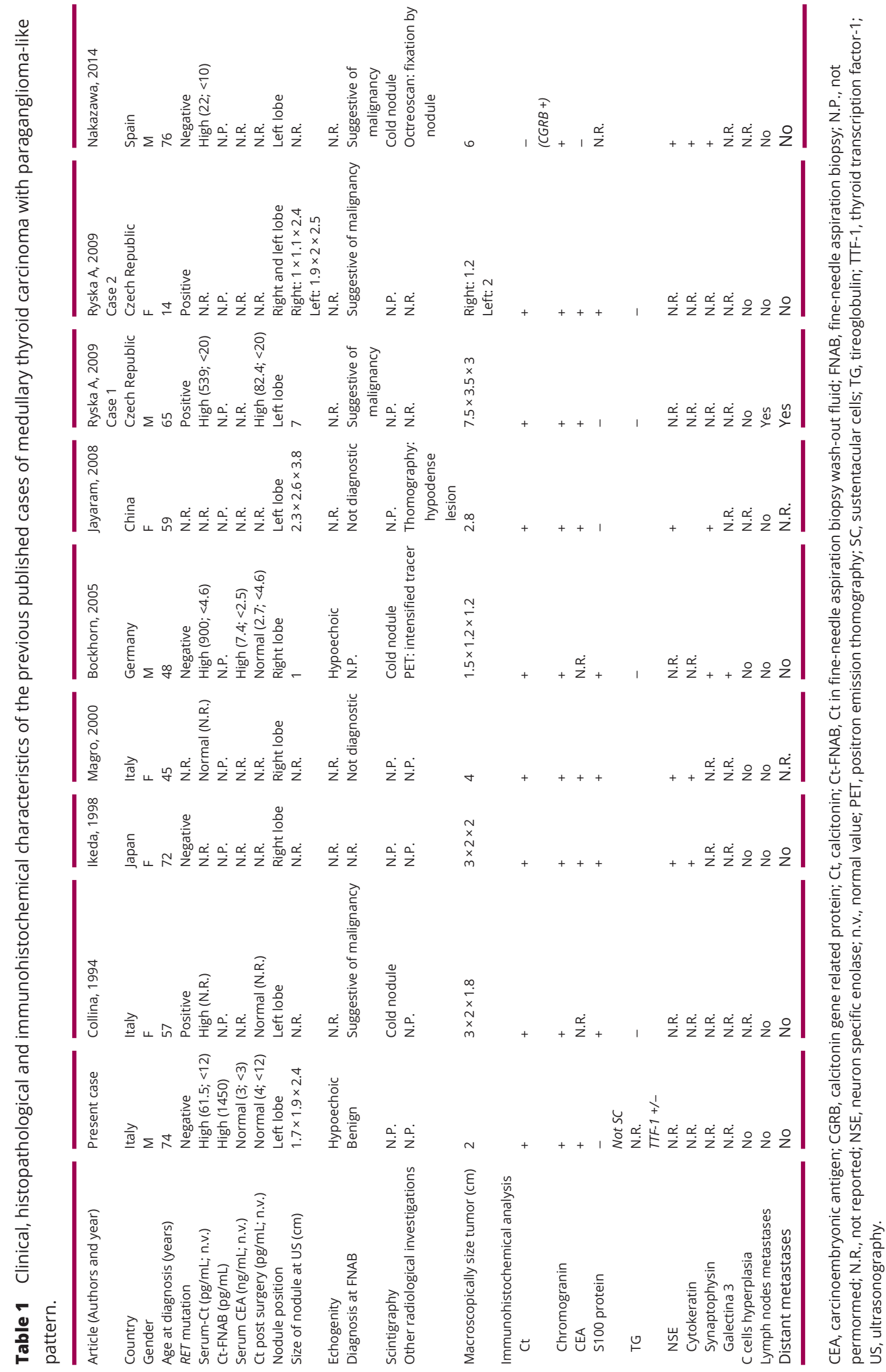


Measurement of $\mathrm{Ct}$ both in serum and in wash-out FNAB fluid could be an additional tool for an early and noninvasive identification of these variants.

Moreover, additional studies are necessary to obtain data of prognoses and predictive factors of this unusual variant of MTC. Despite histological similarity with benign paraganglioma, PG-like MTC is classified as malignant. However, the clinical behavior seems to be indolent, without lymph node involvement or distant metastasis in most cases and with rapid post-surgery $\mathrm{Ct}$ levels fall within normal limits.

\section{Declaration of interest}

The authors declare that there is no conflict of interest that could be perceived as prejudicing the impartiality of this case report.

\section{Funding}

This research did not receive any specific grant from any funding agency in the public, commercial or not-for-profit sector.

\section{Patient consent}

The patient provided written informed consent for the publication of all data and pictures of this case report.

\section{Author contribution statement}

All authors included in this article made substantial contributions to the analysis of the data included, as well as assisted with critical revisions of the writing, and approved the final version for submission for publication. C G, E T and B M participated to the endocrine assessment and treatment of the patient. The authors thank the patient for giving permission for publishing this case.

\section{References}

1 Wells Jr SA, Asa SL, Dralle H, Elisei R, Evans DB, Gagel RF, Lee N, Machens A, Moley JF, Pacini F, et al. Revised American Thyroid Association guidelines for the management of medullary thyroid carcinoma. Thyroid 201525 567-610 (https://doi.org/10.1089/ thy.2014.0335)
2 Cipri C, Vescini F, Torresan F, Pennelli G, Pelizzo MR, Triggiani V, Guastamacchia E \& Grimaldi F. An unusual case of medullary thyroid carcinoma and A revision of current literature. Endocrine, Metabolic and Immune Disorders Drug Targets 201919 226-229 (https://doi.org/ 10.2174/1871530319666181220165350)

3 Collina G, Maiorana A, Fano RA, Cesinaro AM \& Trentini GP. Medullary carcinoma of the thyroid gland with sustentacular cell-like cells in a patient with multiple endocrine neoplasia, type IIA. Report of a case with ultrastructural and immunohistochemical studies. Archives of Pathology and Laboratory Medicine 1994118 1041-1044.

4 Ikeda T, Satoh M, Azuma K, Sawada N \& Mori M. Medullary thyroid carcinoma with a paraganglioma-like pattern and melanin production: a case report with ultrastructural and immunohistochemical studies. Archives of Pathology and Laboratory Medicine 1998122 555-558.

5 Magro G \& Grasso S. Sustentacular cells in sporadic paraganglioma-like medullary thyroid carcinoma: report of a case with diagnostic and histogenetic considerations. Pathology, Research and Practice 2000196 55-59 (https://doi.org/10.1016/ s0344-0338(00)80022-9)

6 Bockhorn M, Sheu SY, Frilling A, Molmenti E, Schmid KW \& Broelsch CE. Paraganglioma-like medullary thyroid carcinoma: a rare entity. Thyroid 200515 1363-1367 (https://doi.org/10.1089/ thy.2005.15.1363)

7 Jayaram G, Hayati JN, Yip CH, Ranganathan S \& Taib NA. Cytologic, histologic and immunocytochemical features in fine needle aspiration of paraganglioma-like variant of medullary carcinoma. Acta Cytologica 200852 119-121 (https://doi. org/10.1159/000325449)

8 Ryska A, Cap J, Vaclavikova E, Dvorakova S, Bendlova B, Hovorkova E \& Kohout A. Paraganglioma-like medullary thyroid carcinoma: fine needle aspiration cytology features with histological correlation. Cytopathology 200920 188-194 (https://doi.org/10.1111/ j.1365-2303.2008.00580.x)

9 Nakazawa T, Cameselle-Teijeiro J, Vinagre J, Soares P, Rousseau E, Eloy C \& Sobrinho-Simões M. C-cell-derived calcitonin-free neuroendocrine carcinoma of the thyroid: the diagnostic importance of CGRP immunoreactivity. International Journal of Surgical Pathology 201422 530-535 (https://doi.org/10.1177/1066896914525228)

10 Trimboli P, Treglia G, Guidobaldi L, Romanelli F, Nigri G, Valabrega S, Sadeghi R, Crescenzi A, Faquin WC, Bongiovanni M, et al. Detection rate of FNA cytology in medullary thyroid carcinoma: a metaanalysis. Clinical Endocrinology 201582 280-285 (https://doi. org/10.1111/cen.12563)

11 Diazzi C, Madeo B, Taliani E, Zirilli L, Romano S, Granata AR, De Santis MC, Simoni M, Cioni K, Carani C, et al. The diagnostic value of calcitonin measurement in wash-out fluid from fine-needle aspiration of thyroid nodules in the diagnosis of medullary thyroid cancer. Endocrine Practice 201319 769-779 (https://doi.org/10.4158/ EP12420.OR)

Received in final form 3 October 2019

Accepted 6 November 2019 